\title{
ÍNDICES DE SANEAMENTO AMBIENTAL EM REGIÕES HIDROGRÁFICAS DO ESTADO DO RIO DE JANEIRO
}

\author{
ENVIRONMENTAL SANITATION INDEXES IN \\ HYDROGRAPHIC REGIONS OF THE RIO DE JANEIRO STATE
}

\author{
Tatiana Lourenço ${ }^{1}$, Rachel Bardy Prado 2 \\ ${ }^{1}$ Instituto Federal de Educação, Ciência e Tecnologia do Rio de Janeiro, Rio de Janeiro, RJ, Brasil. E-mail: \\ tatianaemp@outlook.com \\ 2Embrapa Solos, Rio de Janeiro, RJ, Brasil. E-mail: rachel.prado@embrapa.br
}

Como citar: LOURENÇO, T.; PRADO, R. B. Índices de saneamento ambiental em regiões hidrográficas do estado do Rio de Janeiro. Revista de Gestão de Água da América Latina, v. 16, e7, 2019. 10.21168/rega.v16e7

\begin{abstract}
RESUMO: Muitos são os desafios relacionados ao saneamento ambiental no Brasil, cujos resultados apresentados nos índices, na maioria das vezes, são insatisfatórios em relação aos valores obtidos na média global e aos padrões estabelecidos pela legislação brasileira. No Estado do Rio de Janeiro a situação não é diferente, alcançando para alguns índices uma posição bastante insatisfatória em relação aos demais Estados brasileiros. 0 presente estudo se propôs a obter, aplicar e classificar índices de saneamento ambiental por regiões hidrográficas do Estado do Rio de Janeiro (RHRJ). Para tal foram selecionados 17 indicadores e 10 sub-índices agrupados em 4 categorias: Cobertura, Eficiência, Demanda e Gestão. A partir dos indicadores e referências, foram aplicadas equações específicas e estabelecidas e pontuadas classes para cada sub-índice, sendo as classes: Satisfatório (verde=3), Aceitável (amarelo=2) e Insatisfatório (vermelho=1). Os valores obtidos para os sub-índices foram somados por município e, posteriormente, efetuou-se uma média por RHRJ, obtendo-se o índice final. A última etapa foi realizar um ranqueamento das RHRJ em função do índice final. Observou-se que não há uma tendência de uma RHRJ apresentar todos os sub-índices insatisfatórios ou satisfatórios, eles são variáveis. A RHRJ VI (Lagos São João) foi a que apresentou maior frequência de sub-índices classificados como Satisfatório, com exceção dos sub-índices Perdas na distribuição e Plano municipal de saneamento em que apresentou classificação Insatisfatório. A classe Aceitável esteve mais presente nos sub-índices Coleta de Esgoto e ICMS Ecológico. Já as RHRJ II e IX (Guandu e Baixo Paraíba do Sul e Itabapoana) foram as que apresentaram a maioria dos sub-índices na classe Insatisfatório. 0 método aplicado contribuiu para se ter um panorama integrado da situação de saneamento ambiental nas RHRJ.
\end{abstract}

Palavras-chave: indicadores, classificação, sub-índices, categorias, recursos hídricos.

ABSTRACT: There are many challenges related to environmental sanitation in Brazil, whose results presented in the indices are, in most cases, unsatisfactory in relation to the values obtained in the global average and the standards established by Brazilian legislation. In the State of Rio de Janeiro the situation is not different, reaching for some indices a very unsatisfactory position in relation to the other Brazilian states. The present study proposed to obtain, to apply and to classify indexes of environmental sanitation byhydrographic regions of the State of Rio de Janeiro (RHRJ). To this end, 17 indicators and 10 sub-indexes were grouped into four categories: Coverage, Efficiency, Demand and Management. From the indicators and references, specific equations were applied and classes were established and scored for each sub-index, being: Satisfactory (green=3), Acceptable (yellow=2) and Unsatisfactory (red=1). The values obtained for the sub-indices were summed by municipality and, later, an average was made by RHRJ, obtaining the final index. The last step was to perform a RHRJ ranking according to the final index. It was observed that there is no tendency for an RHRJ to present all sub-indices unsatisfactory or satisfactory, they are variable. RHRJ VI (Lagos São João) presented the highest frequency of sub-indices classified as Satisfactory, with the exception of the sub-indices Losses in the distribution and Municipal sanitation plan in which it presented an Unsatisfactory classification. The Acceptable class was more present in the sub-indexes Sewage Collection and Ecological ICMS. On the other hand, the RHRJ II and IX (Guandu and Baixo Paraíba do Sul and Itabapoana) presented the majority of the

Recebido: 07/01/2019. Revisado: 29/04/2019. Aceito: 03/07/2019. 
sub-indices in the Unsatisfactory class. The applied method contributed to an integrated picture of the situation of environmental sanitation in the RHRJ.

Keywords: indicators, classification, sub-indexes, categories, water resourses.

\section{INTRODUÇÃo}

Foram concluídas em 2015 as negociações que culminaram na adoção dos Objetivos do Desenvolvimento Sustentável (ODS), por ocasião da Cúpula das Nações Unidas para o Desenvolvimento Sustentável. Seguindo mandato da Conferência Rio+20, os ODS deverão orientar as políticas nacionais e as atividades de cooperação internacional nos próximos quinze anos, sucedendo e atualizando os Objetivos do Desenvolvimento do Milênio (ODM) (ORGANIZAÇÃO DAS NAÇÕES UNIDAS, 2015).

Chegou-se a um acordo que contempla 17 Objetivos e 169 metas, envolvendo temáticas diversificadas. 0 Objetivo de Desenvolvimento Sustentável (ODS) 6 da Agenda 2030 se relaciona a "[...] assegurar a disponibilidade e gestão sustentável da água e saneamento para todos" (ORGANIZAÇÃO DAS NAÇÕES UNIDAS, 2015). Ressalta-se que o Brasil participou de todas as sessões da negociação intergovernamental.

O saneamento básico compreende o abastecimento de água potável, coleta e tratamento de esgoto, manejo de resíduos sólidos, manejo de águas pluviais e controle de doenças relacionadas, preocupando-se mais com a questão do acesso ao serviço. Já o saneamento ambiental possui aplicação um pouco mais ampla, além do acesso aos serviços de saneamento, incluem as questões ambientais e de preservação ambiental, visando promover a qualidade e a melhoria do meio ambiente, contribuir para a saúde pública e o bem estar da população (AQUINO, 2018). Tucci (2017) apresenta um histório das tendências do saneamento no mundo, assim como o cenário do Brasil e de outros países em desenvolvimento.

A América Latina, principalmente o Brasil, a Colômbia e o Peru estão entre os dez países do mundo com maior quantidade de água doce. Apesar disso, 106 milhões de latino-americanos ainda não dispõem de banheiro em suas residências e 34 milhões não têm acesso constante a água potável (BANCO MUNDIAL, 2018). Este fato tem sérias implicações na saúde da população. Estudo realizado por Teixeira et al. (2014) no Brasil concluiu que as doenças relacionadas ao saneamento básico inadequado foram responsáveis, em média, por 13.449 óbitos por ano de 2001 a 2009. Conforme Tomeleri, Campos e Morete (2013) o investimento em saneamento ambiental pode reduzir a demanda na área da saúde e, apesar de gerar determinados gastos em um primeiro momento, com o transcorrer do tempo, a situação pode se reverter, pois serão poupados recursos que seriam destinados a solucionar problemas de insalubridade da população por deficiências no saneamento ambiental.

Em 2011, o Instituto Trata Brasil divulgou uma pesquisa em conjunto com o Conselho Empresarial Brasileiro para o Desenvolvimento Sustentável que mostrava o Brasil na 112ª posição no desenvolvimento do saneamento. 0 estudo comparou 200 países e se baseava no IDH para mensurar tal indicador. 0 Brasil, estando entre as dez maiores economias do mundo, ficou atrás de diversos países, como os vizinhos Chile e Argentina.

O problema é grave e embora a questão do saneamento básico afete a todos, as famílias de baixa renda e residentes em áreas irregulares são as mais prejudicadas e que mais sofrem com os seus impactos negativos (INSTITUTO TRATA BRASIL, 2017). Contudo, os investimentos necessários à solucionar o problema são elevadíssimos. Queiroz e Nascimento (2016) fizeram uma estimativa de investimentos em saneamento em Minas Gerais, por exemplo, concluindo que os investimentos teriam que aumentar até 2028 e 2033 em 61\% e 47\%, respectivamente. Desta forma, Souza et al. (2015) propõem um novo olhar sobre a tríade 'desenvolvimento, ambiente e saúde', com o objetivo de formular estratégias inovadoras para garantir o acesso mais amplo ao saneamento.

Também se destaca no Brasil o índice de perdas de água nas redes de abastecimento. Em grandes números, os dados do Sistema Nacional de Informações sobre Saneamento (BRASIL, 2014) mostram que as perdas na distribuição equivalem à $37 \%$ e que as perdas financeiras totais equivalem à 39\%, não entrando na receita do setor. Estimou-se que a água não faturada pelas empresas foi de 6,53 bilhões de $\mathrm{m}^{3}$ de água tratada, perfazendo perda financeira de $\mathrm{R} \$ 8,01$ bilhões ao ano. Tais perdas equivalem a cerca de $80 \%$ dos investimentos em água e esgoto realizados em 2013. Na projeção do estudo, se em cinco anos houvesse uma queda de 15\% nas perdas no Brasil, ou seja, de 39\% para 33\%, os ganhos totais acumulados em relação ao ano inicial seriam da ordem de R \$3,85 bilhões (INSTITUTO TRATA BRASIL, 2017).

No Estado do Rio de Janeiro a situação não é diferente e muitos são os desafios. No ranqueamento do saneamento em 2017 das 100 maiores cidades brasileiras, alguns municípios do RJ como São Gonçalo, São João de Meriti, Nova Iguaçu e Duque de Caxias ficaram nas últimas 20 posições. Os problemas relativos ao saneamento ambiental do estado do Rio de Janeiro se relacionam ao tipo e condição dos aterros sanitários, aos índices de cobertura de coleta e tratamento de esgoto e de água, 
às perdas nos sistemas de abastecimento de água, perpassando pela gestão dos recursos hídricos, planejamento de ações mitigadoras e investimentos para assegurar maior qualidade do saneamento ambiental, realizado pelo estado (INSTITUTO TRATA BRASIL, 2017).

Para avaliar estes aspectos, geralmente são utilizados indicadores e índices de qualidade ambiental. Um indicador pode ser definido como um parâmetro ou um valor derivado de parâmetros que fornece informações sobre um determinado fenômeno (ORGANISATION FOR ECONOMIC COOPERATION AND DEVELOPMENT, 1993). Segundo Rufino (2005) um índice ambiental é uma classificação numérica ou descritiva de um grande volume de informação ambiental, cujo objetivo é realizar a simplificação desses dados facilitando a tomada de decisão relativa à questão ambiental sob análise. Ainda segundo este autor para a composição de um índice algumas características são desejáveis: facilidade de uso; deve refletir o conjunto de informações contidas nos indicadores que o compõem, principalmente refletirem as alterações e pesos destes indicadores; não deve ser ambíguo, e; deve permitir a determinação de tendências no espaço e no tempo. Giovannini et al. (2008) elaboraram um manual para a construção de indicadores compostos.

Para Schwemlein, Cronk e Bartram (2016), do ponto de vista da administração pública, o monitoramento de dados é importante e necessário para acompanhar o andamento das atividades, melhorar a transparência da contabilidade e demonstrar resultados. No contexto internacional, há uma crescente utilização de indicadores de desempenho no setor de saneamento para monitorar o fornecimento, a regulamentação e o planejamento desses serviços (SPERLING; SPERLING, 2013). Uma ferramenta que tem sido usada frequentemente para influenciar as discussões políticas nacionais e internacionais é o ranking das cidades verdes (MEIJERING; KERN; TOBI, 2014).

No Brasil, há duas iniciativas relevantes, o ranking de saneamento básico das 100 maiores cidades, pelo Instituto Trata Brasil (2017) e pelo Programa Municipal Verde Azul dentro da Secretaria do Meio Ambiente do Estado de São Paulo (SÃO PAULO, 2019). Há também uma iniciativa de classificação de saneamento que foi realizada pelo governo da Índia. 0 que esses três rankings têm em comum é que eles são baseados em indicadores ou índices que são aplicados às cidades e que variam em número de 12 a 70. No entanto, não há rankings ou iniciativas estruturadas para elaborar indicadores brasileiros que contemplem os componentes básicos saneamento de acordo com a Lei $\mathrm{n}^{\mathrm{o}} 11.445$ / 2007, a fim de medir e monitorar o saneamento adequado (MEIJERING; KERN; TOBI, 2014).

Van Bellen (2004) fez um levantamento dos principais sistemas de avaliação de indicadores de sustentabilidade. Lima (2013) utilizou o saneamento ambiental como indicador de análise da qualidade ambiental urbana em Presidente Epitácio, SP. Tucci (2017) apresenta alguns exemplos de indicadores de saneamento, assim como um indicador de sustentabilidade hídrica urbana. Também Nirazawa e Oliveira (2018) analisaram variáveis para elaboração de indicadores de saneamento básico para os municípios paulistas a fim de gerar informações para a administração pública.

Contudo, Dias, Gomes e Alkmim (2011) mencionam que no Brasil ainda existem poucos estudos que se propõem a analisar, de forma integrada e por bacia hidrográfica, os componentes do ambiente com o objetivo de diagnosticar e propor melhorias no saneamento ambiental. Os trabalhos existentes foram desenvolvidos por órgãos governamentais responsáveis pelo monitoramento de componentes ambientais, como é o caso da Companhia Ambiental do Estado de São Paulo (CETESB). Estes estudos lidam com a dificuldade de se encontrar bases de dados sistematizadas, acessíveis e em escala adequada. Para Braga et al. (2004) um outro desafio é a seleção e ponderação dos indicadores e composição dos índices, pois exigem o conhecimento de diversos especialistas, sendo os pesos muitas vezes subjetivos.

No contexto apresentado foi realizado o presente estudo, visando obter, aplicar e classificar índices de saneamento ambiental para as regiões hidrográficas do estado do Rio de Janeiro, levando em conta que a gestão de recursos hídricos ocorre no nível das bacias hidrográficas.

\section{MATERIAL E MÉTODOS}

\section{Levantamento e sistematização dos dados}

Inicialmente foram levantados dados a respeito de aspectos do saneamento ambiental dos municípios do e agrupados de acordo com as regiões hidrográficas do estado do Rio de Janeiro estabelecidas por INEA (RIO DE JANEIRO, 2013) (Figura 1). 


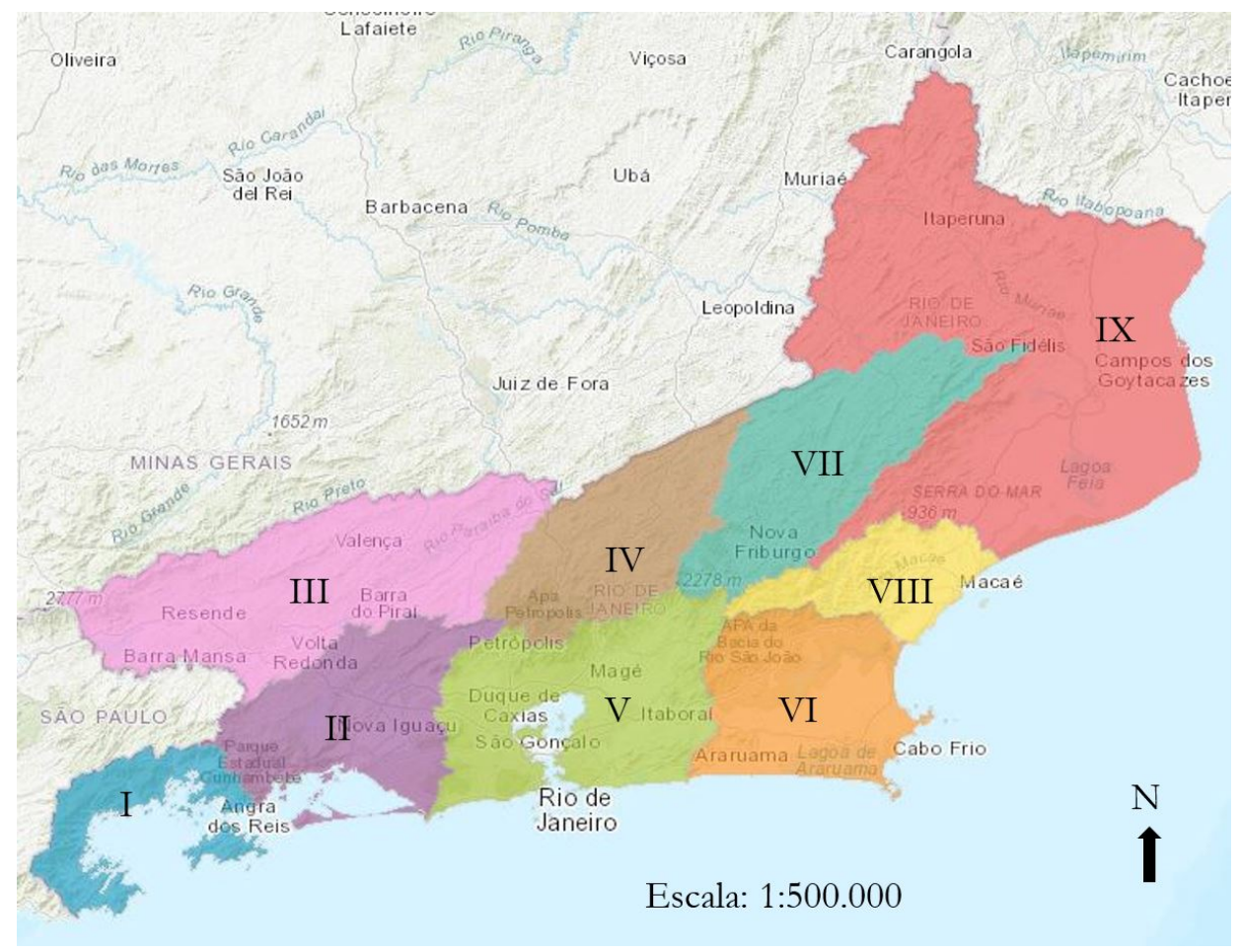

Figura 1. Regiões hidrográficas do estado do Rio de Janeiro.

Fonte: Adaptado de GEOINEA (2019).

Foram considerados para cada região hidrográfica os municípios cuja área estava predominantemente contida na mesma, isto é, cada município somente foi contabilizado em uma RHRJ, para evitar sobreposição de informação (Tabela 1).

Tabela 1. Regiões hidrográficas e seus respectivos municípios, no Estado do Rio de Janeiro.

\begin{tabular}{|c|c|}
\hline Regiões hidrográficas & Municípios \\
\hline Região hidrográfica I: Baía de Ilha Grande & Angra dos Reis e Paraty \\
\hline Região hidrográfica II: Guandu & $\begin{array}{l}\text { Engenheiro Paulo de Frontin, Itaguaí, Japeri, Mangaratiba, } \\
\text { Mendes, Paracambi, Piraí, Queimados, Rio Claro e Seropédica }\end{array}$ \\
\hline $\begin{array}{l}\text { Região hidrográfica III: Médio Paraíba do } \\
\text { Sul }\end{array}$ & $\begin{array}{l}\text { Barra do Piraí, Barra Mansa, Comendador Levy Gasparian, Itatiaia, } \\
\text { Pinheiral, Porto Real, Quatis, Resende, Rio das Flores, Valença, } \\
\text { Volta Redonda, Miguel Pereira, Paraíba do Sul, Paty do Alferes, } \\
\text { Três Rios e Vassouras }\end{array}$ \\
\hline Região hidrográfica IV: Piabanha & $\begin{array}{l}\text { Areal, Carmo, Petrópolis, São José do Vale do Rio Preto, Sapucaia, } \\
\text { Sumidouro e Teresópolis }\end{array}$ \\
\hline Região hidrográfica V: Baía de Guanabara & $\begin{array}{l}\text { Belford Roxo, Cachoeiras de Macacu, Duque de Caxias, } \\
\text { Guapimirim, Itaboraí, Magé, Maricá, Mesquita, Nilópolis, Niterói, } \\
\text { Nova Iguaçu, Rio Bonito, Rio de Janeiro, São Gonçalo, São João de } \\
\text { Meriti e Tanguá }\end{array}$ \\
\hline Região hidrográfica VI: Lagos São João & $\begin{array}{l}\text { Araruama, Armação dos Búzios, Arraial do Cabo, Cabo Frio, } \\
\text { Casimiro de Abreu, Iguaba Grande, São Pedro da Aldeia, } \\
\text { Saquarema, e Silva Jardim }\end{array}$ \\
\hline Região hidrográfica VII: Rio Dois Rios & $\begin{array}{l}\text { Bom Jardim, Cantagalo, Cordeiro, Duas Barras, Itaocara, Macuco, } \\
\text { Nova Friburgo, Santa Maria Madalena, São Fidélis e São Sebastião } \\
\text { do Alto }\end{array}$ \\
\hline $\begin{array}{l}\text { Região hidrográfica VIII: Macaé e das } \\
\text { Ostras }\end{array}$ & Macaé e Rio das Ostras \\
\hline $\begin{array}{l}\text { Região hidrográfica IX: Baixo Paraíba do } \\
\text { Sul e Itabapoana }\end{array}$ & $\begin{array}{l}\text { Aperibé, Bom Jesus, Cambuci, Campos dos Goytacazes, Carapebus, } \\
\text { Cardoso Moreira, Conceição de Macabu, Italva, Itaperuna, Laje do } \\
\text { Muriaé, Miracema, Natividade, Porciúncula, Quissamã, Santo } \\
\text { Antônio de Pádua, São Francisco de Itabapoana, São João da Barra, } \\
\text { São José de Ubá, Trajano de Morais e Varre-Sai }\end{array}$ \\
\hline
\end{tabular}




\section{Composição dos sub-índices}

Inicialmente foram estabelecidas quatro categorias para a composição dos sub-índices relacionados ao saneamento ambiental, a saber: Cobertura, Eficiência, Demanda e Gestão. Entende-se por Cobertura o atendimento à população dos municípios estudados em termos de serviços de água e esgoto; pela Eficiência entende-se a qualidade dos serviços prestados em termos de água e esgoto; pela Demanda entende-se a necessidade de água para suprimento doméstico dos habitantes dos municípios estudados e em termos de Gestão entende-se que inclua ações, instrumentos e políticas que visem o planejamento, a regulação, a conservação e a distribuição justa e sustentável da água à população.

Os índices obtidos nesta etapa do estudo foram denominados de sub-índices, uma vez que se pretendeu também obter um Índice Final de saneamento ambiental para as RHRJ, a partir da integração dos sub-índices. Foram selecionados 17 indicadores na literatura, já comumente aplicados para avaliação de diversos aspectos do saneamento ambiental, que compuseram 10 sub-índices relacionados às quatro categorias. Os indicadores e sub-índices foram selecionados e obtidos em função da disponibilidade de dados necessários à sua aplicação para todos os municípios do Estado RJ, assim como pela sua relevância em medir a situação do saneamento ambiental e impactos de ações em prol de sua eficiência nas diferentes categorias Cobertura, Eficiência, Demanda e Gestão.

Os indicadores selecionados, bem como as equações utilizadas para compor os sub-índices se encontram na Tabela 2 .

Tabela 2. Composição dos sub-índices relativos ao saneamento ambiental nas RHRJ, por categoria.

\begin{tabular}{|c|c|c|c|c|}
\hline Categoria & Sub-índices & Referências & Equação/indicadores & Classificação \\
\hline \multirow{3}{*}{$\begin{array}{l}C \\
O \\
B \\
E \\
R \\
T \\
U \\
R \\
A\end{array}$} & $\begin{array}{l}\text { 1-IN055 - Atendimento } \\
\text { total de água (\%) }\end{array}$ & $\begin{array}{l}\text { Em função da } \\
\text { meta estipulada } \\
\text { (100\%) pelo } \\
\text { BNDES } 1 \\
\text { (PIMENTEL et } \\
\text { al., 2017) }\end{array}$ & $\frac{A G 001}{G E 12 a} \times 100$ & $\begin{array}{l}\text { 90\%-100\%: } \\
\text { Satisfatório } \\
\text { 80\%-90\%: } \\
\text { Aceitável } \\
\text { < 80\%: } \\
\text { Insatisfatório }\end{array}$ \\
\hline & $\begin{array}{l}\text { 2-IN015 - Coleta de } \\
\text { esgoto (\%) }\end{array}$ & $\begin{array}{l}\text { Pacto pelo } \\
\text { Saneamento- } \\
\text { Rio de Janeiro } \\
\quad \text { (2011) }\end{array}$ & $\frac{E S 005}{A G 010-A G 019} \times 100$ & $\begin{array}{l}\text { > 80\%: } \\
\text { Satisfatório } \\
\text { 40\% 80\%: } \\
\text { Aceitável } \\
\text { < 40\%: } \\
\text { Insatisfatório }\end{array}$ \\
\hline & $\begin{array}{c}\text { 3-IN016 - Tratamento } \\
\text { de esgoto (\%) }\end{array}$ & $\begin{array}{c}\text { PLANSAB } \\
(B R A S I L, 2013)^{2}\end{array}$ & $\begin{array}{c}\frac{(E S 006+E S 014+E S 015)}{E S 005+E S 013} x \\
100\end{array}$ & $\begin{array}{c}\text { >63\%: } \\
\text { Satisfatório } \\
\text { 35\%-63\%: } \\
\text { Aceitável } \\
<35 \% \text { : } \\
\text { Insatisfatório } \\
\end{array}$ \\
\hline \multirow{3}{*}{$\begin{array}{l}E \\
F \\
I \\
C \\
I \\
\hat{E} \\
N \\
C \\
I \\
A\end{array}$} & $\begin{array}{l}\text { 4-IN046 - Esgoto } \\
\text { tratado referido à água } \\
\text { consumida (\%) }\end{array}$ & $\begin{array}{l}\text { Costa et al. } \\
\quad \text { (2013) }\end{array}$ & $\frac{E S 006}{A G 010} \times 100$ & 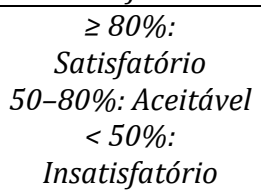 \\
\hline & $\begin{array}{l}\text { 5-IN084 - Incidência } \\
\text { de coliformes fora do } \\
\text { padrão (\%) }\end{array}$ & $\begin{array}{c}\text { Ministério da } \\
\text { Saúde (BRASIL, } \\
\text { 2011) } \\
\end{array}$ & $\frac{Q D 027}{Q D 026} \times 100$ & $\begin{array}{c}\text { 5 5\%: Satisfatório } \\
>\text { 5\%: } \\
\text { Insatisfatório }\end{array}$ \\
\hline & $\begin{array}{l}\text { 6-IN049 - Perdas na } \\
\text { distribuição (\%) }\end{array}$ & $\begin{array}{c}\text { PLANSAB } \\
(\text { BRASIL, 2013)2 }\end{array}$ & $\begin{array}{c}\frac{[(A G 006-A G 024)-A G 010]}{A G 006-A G 024} \\
\times 100\end{array}$ & $\begin{array}{c}\text { <25\%: } \\
\text { Satisfatório } \\
\text { 25\%-37,5\%: } \\
\text { Aceitável } \\
>37,5 \%: \\
\text { Insatisfatório }\end{array}$ \\
\hline $\begin{array}{c}D \\
E \\
M \\
A \\
N \\
D \\
A\end{array}$ & $\begin{array}{l}\text { 7-Nível do tratamento } \\
\text { da água (primário, } \\
\text { secundário, terciário) }\end{array}$ & $\begin{array}{l}\text { Centro Estadual } \\
\text { de Estatísticas, } \\
\text { Pesquisas e } \\
\text { Formação de } \\
\text { Servidores } \\
\text { Públicos do Rio } \\
\text { de Janeiro } \\
(2018)^{3}\end{array}$ & $\begin{array}{l}\text { Média aplicada para a } \\
\text { classificação das RHRJ }\end{array}$ & $\begin{array}{c}\text { 2 2,78: Satisfatório } \\
\text { 1,39-2,78: } \\
\text { Aceitável } \\
<1,39: \\
\text { Insatisfatório }\end{array}$ \\
\hline
\end{tabular}




\begin{tabular}{|c|c|c|c|c|}
\hline & $\begin{array}{c}\text { 8-IN022 - Consumo } \\
\text { médio de água per } \\
\text { capita } \\
\text { (litro/habitante/dia) }\end{array}$ & $\begin{array}{l}\text { ORGANIZAÇÃO } \\
\text { DAS NAÇÕES } \\
\text { UNIDAS }(2015)^{4}\end{array}$ & $\frac{A G 010}{A G 001} \times 1.000 .000 / 365$ & $\begin{array}{c}\text { <110 lit./hab./dia: } \\
\text { Satisfatório } \\
\text { 110-165: Aceitável } \\
>165 \text { lit./hab./dia: } \\
\text { Insatisfatório }\end{array}$ \\
\hline & $\begin{array}{c}\text { 9- Plano Municipal de } \\
\text { Saneamento }\end{array}$ & $\begin{array}{c}\text { PLANSAB } \\
(B R A S I L, 2013)^{2}\end{array}$ & $\frac{N I}{N T} \times 100$ & $\begin{array}{c}\geq 37 \%: \\
\text { Satisfatório } \\
\text { 37\%-18,5: } \\
\text { Aceitável } \\
<18,5: \\
\text { Insatisfatório }\end{array}$ \\
\hline $\begin{array}{l}G \\
E \\
S \\
T \\
\tilde{A} \\
O\end{array}$ & $\begin{array}{l}\text { 10-ICMS-E (relativo ao } \\
\text { tratamento de esgoto) }\end{array}$ & $\begin{array}{c}\text { Em função dos } \\
\text { valores } \\
\text { repassados aos } \\
\text { municípios do RJ } \\
\text { no ano de } 2016 \\
\text { (CENTRO } \\
\text { ESTADUAL DE } \\
\text { ESTATÍSTICAS, } \\
\text { PESQUISAS E } \\
\text { FORMAÇÃO DE } \\
\text { SERVIDORES } \\
\text { PÚBLICOS DO } \\
\text { RIO DE JANEIRO, } \\
\text { 2018) }\end{array}$ & $\frac{V 1}{V 2} \times 100$ & $\begin{array}{c}>50 \%- \\
\text { Satisfatório } \\
\text { 10\%-50\%: } \\
\text { Aceitável 0-10\%: } \\
\text { Insatisfatório }\end{array}$ \\
\hline
\end{tabular}

Legenda das referências: ${ }^{1}$ Banco Nacional do Desenvolvimento; ${ }^{2}$ Plano Nacional de Saneamento Básico; ${ }^{3}$ Centro Estadual de Estatísticas, Pesquisa e Formação de Servidores Públicos do Rio de Janeiro; ${ }^{4}$ Organização das Nações Unidas.

Legenda dos indicadores: AG001: População total atendida com abastecimento de água; GE12a: População residente total, segundo o IBGE; ES005: Volume de esgotos coletado; ES006: Volume de esgotos tratado; ES013: Volume de esgotos bruto importado; ES014: Volume de esgoto importado tratado nas instalações do importador; ES015: Volume de esgoto bruto exportado tratado nas instalações do exportador; AG006: Volume de água produzido; AG010: Volume de água consumido; AG019: Volume de água tratada exportada; AG024: Volume de serviço (valor da soma dos volumes anuais de água usados para atividades operacionais e especiais); QD026: Quantidade de amostras para coliformes totais analisadas; QD027: Quantidade de amostras para coliformes totais com resultados fora do padrão; NI: Quantidade de municípios que possuem Plano Municipal de Saneamento; NT: Quantidade total de municípios que compõem a região hidrográfica; V1: Valor proveniente do tratamento de esgoto referente a cada município da Região Hidrográfica em 2016; V2: Valor total arrecadado por cada município da região hidrográfica em 2016.

A maioria dos dados utilizados foi obtida por meio do Sistema Nacional de Informações sobre Saneamento (SNIS), elaborado no âmbito do Programa de Modernização do Setor de Saneamento (PMSS), vinculado à Secretaria Nacional de Saneamento Ambiental do Ministério das Cidades (BRASIL, 2014). As informações compiladas pelo Ministério das Cidades possuem dois anos de defasagem, de maneira que os dados utilizados no presente artigo são referentes ao ano de 2015. Para os sub-índices de água e esgoto idênticos aos utilizados pelo SNIS, manteve-se o seu código identificado por IN na Tabela 2.

Também foram utilizados dados do ICMS Ecológico (ICMS-E) obtidos no Centro Estadual de Estatísticas, Pesquisas e Formação de Servidores Públicos do Rio de Janeiro (2018). O ICMS Ecológico é um mecanismo tributário que possibilita aos municípios acesso a parcelas maiores que àquelas que já têm direito, dos recursos financeiros arrecadados pelos estados através do Imposto sobre Circulação de Mercadorias e Serviços, o ICMS, em razão do atendimento de determinados critérios ambientais estabelecidos em leis estaduais. Não é um novo imposto, mas sim a introdução de novos critérios de redistribuição de recursos do ICMS, que reflete o nível da atividade econômica nos municípios, em conjunto com a preservação e conservação do meio ambiente.

\section{Classificação dos resultados dos sub-índices e obtenção do índice final e ranqueamento do saneamento ambiental para as RHRJ}

Foram estabelecidas classes para cada sub-índice, de acordo com referências obtidas na literatura, sendo elas denominadas de: Satisfatório (verde), Aceitável (amarelo) e Insatisfatório (vermelho). No caso do sub-índice IN084 - Incidência de coliformes fora do padrão (\%), somente foram utilizadas duas classes: Satisfatório e Insatisfatório, pelo fato da referência de limites utilizada (BRASIL, 2011) já preconizar apenas duas classes. A Tabela 2 apresenta as referências que subsidiaram o estabelecimento dos limites dos intervalos das classes para cada sub-índice. 
Para a obtenção do Índice Final de saneamento ambiental para cada RHRJ, as classes obtiveram uma pontuação Satisfatório ( 3 pontos), Aceitável ( 2 pontos) e Insatisfatório (1 ponto) para cada sub-índice. A partir de então os sub-índices foram somados para cada município, depois para a RHRJ e finalmente este somatório foi dividido pelo número de municípios integrantes de cada RHRJ. A partir de então foi realizado um ranqueamento das RHRJ em função da pontuação obtida no índice final, sendo que a maior pontuação significava melhor condição de saneamento ambiental, sendo o 1ํ lugar a situação melhor e o 9o lugar a situação pior.

\section{RESULTADOS E DISCUSSÃO}

\section{Sub-índices de saneamento ambiental para as RHRJ}

Os resultados são apresentados em relação aos municípios, mas de forma composta para cada região hidrográfica. Obteve-se na categoria Cobertura em relação ao sub-índice 1: Atendimento total de água (\%) que apenas a RHRJ VI (Lagos São João) apresentou classe Satisfatório; as RHRJ III, VII e VIII (Médio Paraíba do Sul, Rio Dois Rios e Macaé e das Ostras) apresentaram classe Aceitável e as RHRJ I, II, IV, V e IX (Baía de Ilha Grande, Guandu, Piabanha, Baía de Guanabara e Baixo Paraíba do Sul e Itabapoana) apresentaram classe Insatisfatório (Figura 2(1)). Em relação ao sub-índice 2: Coleta de esgoto (\%) - segundo Plano Estadual de Recursos Hídricos - PERHI (RIO DE JANEIRO, 2018), a maior parte das RHRJ tem mais de $50 \%$ da população atendida com rede coletora de esgotos. Neste estudo obteve-se que nenhuma RHRJ foi classificada como Satisfatório; apenas a RHRJ VIII (Macaé e das Ostras) foi classificada como Insatisfatório e as demais RHRJ foram classificadas como Aceitável (Figura 2(2)). Em relação ao sub-índice 3: Tratamento de esgoto (\%) as RHRJ I, III, VI e VIII (Baía de Ilha Grande, Médio Paraíba do Sul, Lagos São João e Macaé e das Ostras) apresentaram classificação Satisfatório; as RHRJ IV e V (Piabanha e Baía de Guanabara) apresentaram classificação Aceitável e as RHRJ II, VII e IX (Guandu, Rio Dois Rios e Baixo Paraíba do Sul e Itabapoana) apresentaram classe Insatisfatório (Figura 2(3)).

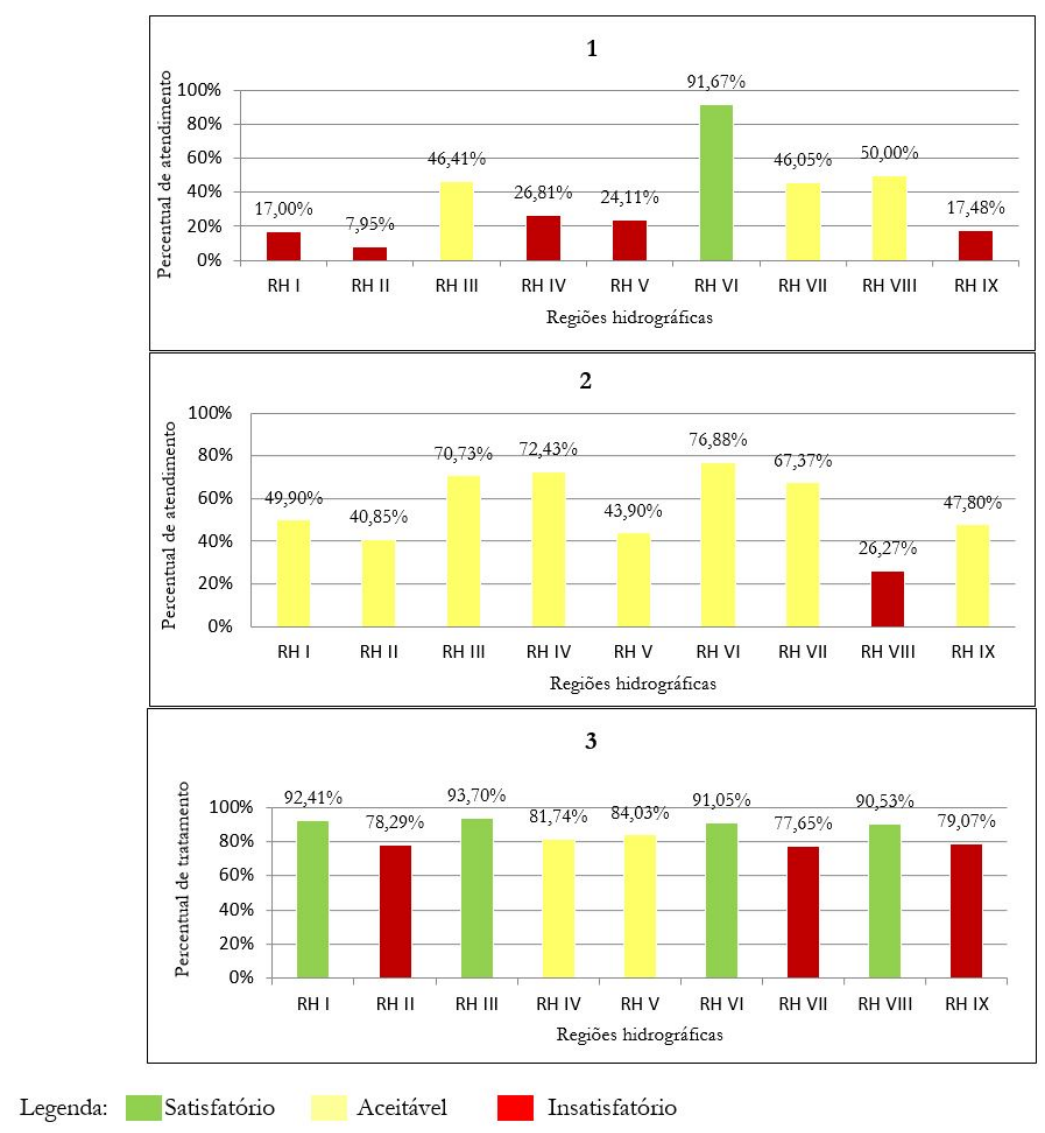

Figura 2. Sub-índices da categoria Cobertura: 1 - Atendimento total de água (\%), 2 - Coleta de esgoto (\%) e 3 - Tratamento de esgoto (\%). 
Em relação à categoria Eficiência obteve-se para o sub-índice 4: Esgoto tratado referido à água consumida (\%) que nenhuma RHRJ foi classificada como Satisfatório, apenas a RHRJ VI (Lagos São João) apresentou classe Aceitável e o restante das RHRJ ficou na classe Insatisfatório (Figura 3(4)). Em relação ao sub-índice 5: Incidência de coliformes fora do padrão (\%) estabelecido para a potabilidade da água para o abastecimento humano, a RHRJ I, II e VIII (Baía de Ilha Grande, Guandu e Macaé e das Ostras) obtiveram classificação Insatisfatório e as demais obtiveram classificação Satisfatório, sendo que as RHRJ VI, VII e IX (Lagos São João, Rio Dois Rios e Baixo Paraíba do Sul e Itabapoana) apresentaram ausência de coliformes (Figura 3(5)).

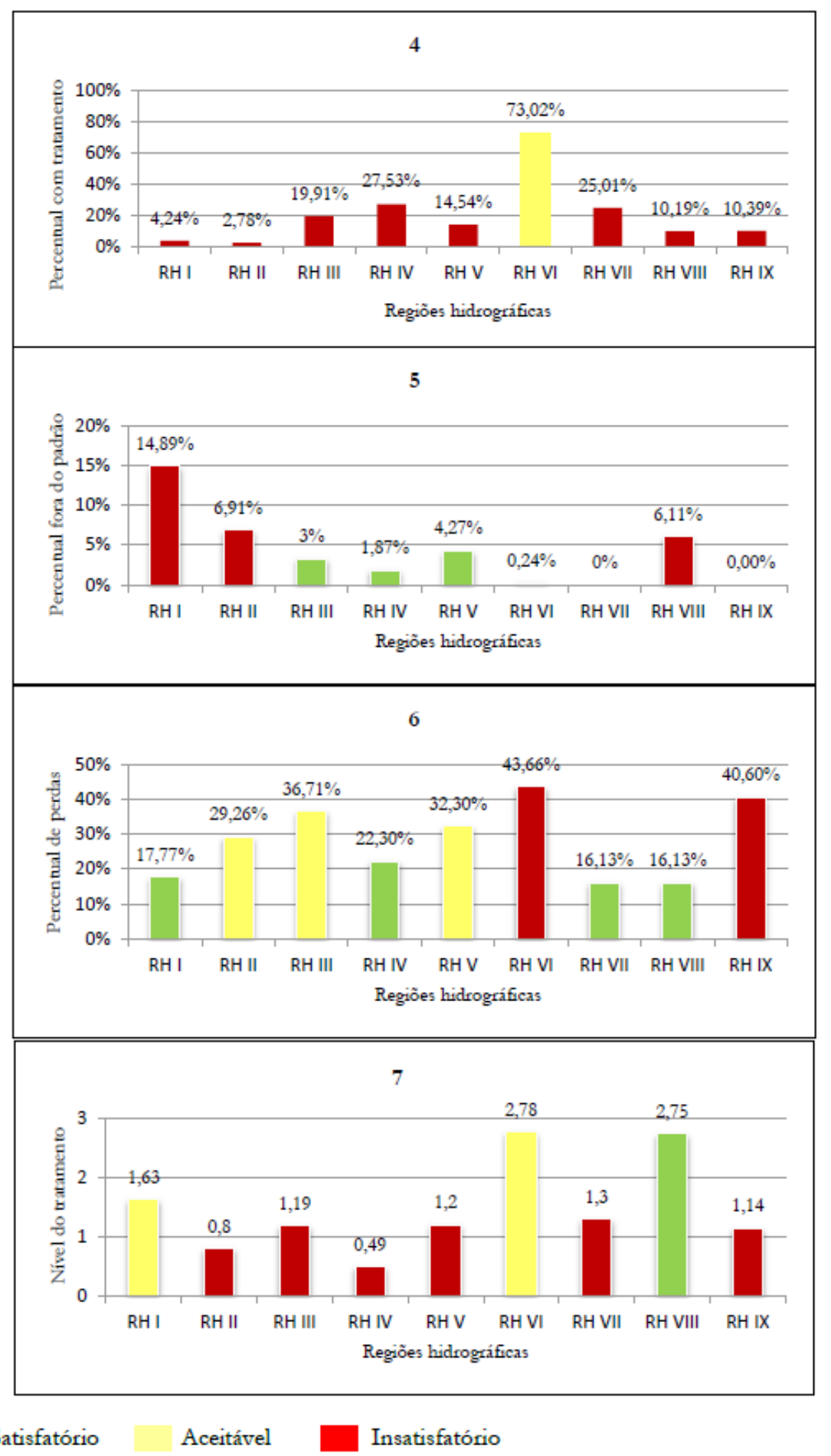

Figura 3. Sub-índices da categoria Eficiência: 4 - Esgoto tratado referido à água consumida, 5 - Incidência de coliformes fora do padrão (\%), 6 - Perdas de água na distribuição (\%), 7 - Nível do tratamento da água. 
No caso das RHRJ VI, VII e IX (Lagos São João, Rio Dois Rios e baixo paraíba do sul e Itabapoana) este sub-índice apresentou alguns valores tão baixos que nem apareceram na Figura 3(5), devido à escala gráfica. A presença de coliformes na água ocorre devido à falta de investimentos em tratamento de esgotos, mas também pelo tipo de tratamento empregado nas estações instaladas, que não apresentam eficiência de remoção suficiente para reduzir as contagens bacteriológicas, de maneira a permitir a adequação dos níveis de acordo com os padrões de enquadramento (GONÇALVES, 2016).

No sub-índice 6: Perdas de água na distribuição (\%) as RHRJ I, IV, VII e VIII (Baía de Ilha Grande, Piabanha, Rio Dois Rios e Macaé e das Ostras) apresentaram classe Satisfatório; as RHRJ II, III e V (Guandu, Médio Paraíba do Sul e Baia de Guanabara) apresentaram classe Aceitável e as RHRJ VI e IX (Lagos São João e Baixo Paraíba do Sul e Itabapoana) apresentaram classe Insatisfatório (Figura 3(6)). Em relação ao sub-índice 7: Nível do tratamento da água para abastecimento, apenas a RHRJ VIII (Macaé e das Ostras) obteve classificação Satisfatória; as RHRJ I e VI (Baía de Ilha Grande e Lagos São João) foram classificadas como Aceitável e a maioria das RHRJ foi classificada como Insatisfatório (Figura 3(7)).

Em relação à categoria Demanda obteve-se para o sub-índice 8: Consumo médio de água per capta, que todas as RHRJ apresentaram valores abaixo do que a ONU recomenda (110 l/hab./dia), ficando na classe Insatisfatório, o que significa menor demanda per capta de água para abastecimento, com exceção da RHRJ VI (Lagos São João) que apresentou classificação Aceitável (Figura 4(8)).

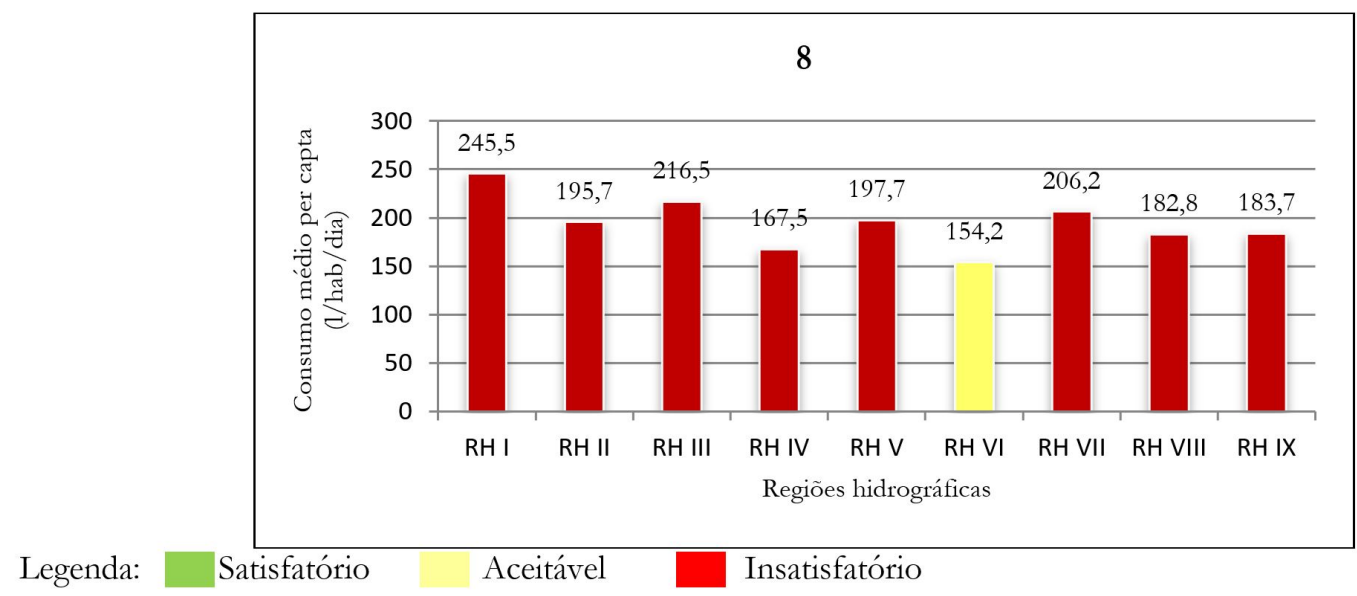

Figura 4. Sub-índice da categoria Demanda: 8-Consumo médio de água per capita (l/hab/dia).

A respeito da categoria Gestão pode-se dizer em relação ao sub-índice 9: Plano municipal de saneamento, que a RHRJ VII (Rio Dois Rios) recebeu classificação Satisfatório, a RHRJ IX (Baixo Paraíba do Sul e Itabapoana) recebeu classificação Aceitável e as demais receberam classificação Insatisfatório, sendo que algumas RHRJ não possuíam nenhum município com plano municipal de saneamento, por isto apresentaram 0\% (Figura 5(9)). No caso das RHRJ I, V, VI e VIII (Baía de Ilha Grande, Baía de Guanabara, Lagos São João e Macaé e das Ostras) este sub-índice apresentou valores tão baixos que nem apareceram na Figura 5(9), devido à escala gráfica. Quanto ao sub-índice 10: ICMS Ecológico, relativo ao tratamento de esgoto, obteve-se que a RHRJ VI (Lagos São João) apresentou classificação Satisfatório; as RHRJ I, IV e V (Baía de Ilha Grande, Piabanha, Baía de Guanabara) apresentaram classificação Insatisfatório e as demais apresentaram classificação Aceitável (Figura 5(10)). No caso da RHRJ II (Médio Paraíba do Sul) este sub-índice apresentou alguns valores tão baixos que nem apareceram na Figura 5(10), devido à escala gráfica. 

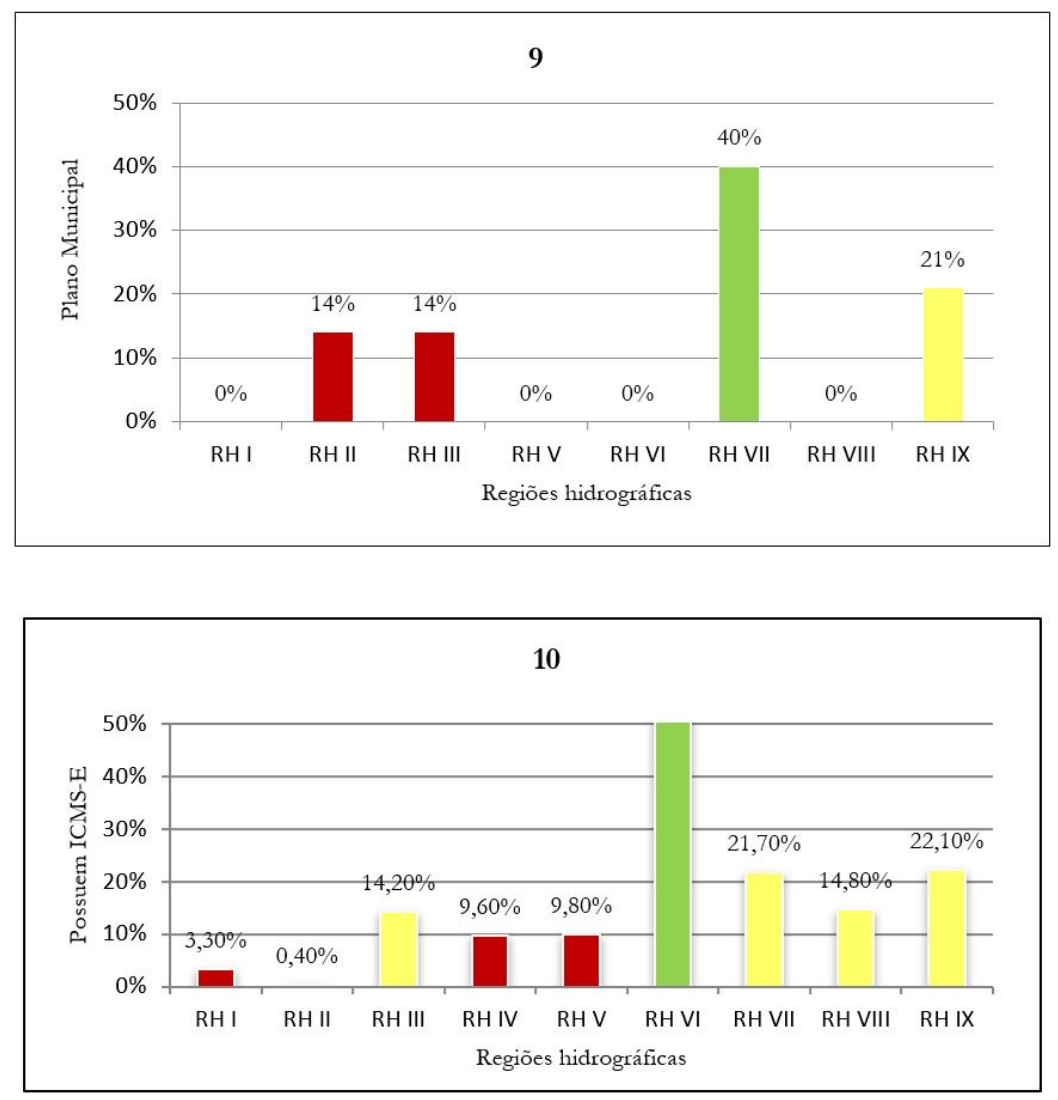

Legenda: Satisfatório Aceitável Insatisfatório

Figura 5. Sub-índices da categoria Gestão: 9 - Plano municipal de saneamento e 10 - ICMS Ecológico relativo ao tratamento de esgoto.

A RHRJ VI (Lagos São João) foi a que apresentou maior frequência de sub-índices classificados como Satisfatório, com exceção dos sub-índices Perdas na distribuição e Plano municipal de saneamento em que apresentou classificação Insatisfatório. A classe Aceitável esteve mais presente nos sub-índices Coleta de Esgoto e ICMS Ecológico. Já as RHRJ II e IX (Guandu e Baixo Paraíba do Sul e Itabapoana) foram as que apresentaram a maioria dos sub-índices na classe Insatisfatório. No caso da RHRJ IX (Baixo Paraíba do Sul) isto se deve provavelmente ao fato de estar na porção mais plana do estado do Rio de Janeiro, recebendo as contribuições de esgotos provindos de toda a área da bacia do rio Paraíba do Sul, bem como recebe sedimentos advindos dos processos erosivos pela degradação das pastagens nesta região.

Kury et al. (2008) ao avaliar a percepção da população residente nesta RHRJ IX em relação à qualidade da água, obtiveram que a maioria dos entrevistados não está satisfeita com a atual situação do rio Paraíba do Sul e acreditam que a atividade industrial e o esgoto doméstico são seus principais agentes poluidores, estando disposta a contribuir com algum aumento no valor de produtos manufaturados e alimentos para melhorias na qualidade de suas águas.

De acordo com os estudos desenvolvidos pela Associação pró-Gestão das Águas da Bacia Hidrográfica do Rio Paraíba do Sul (2014) foram diagnosticadas que as principais fontes de poluição pontuais e difusas na bacia correspondem às cargas orgânicas pelo lançamento de esgotos sanitários, chorume produzido nos aterros, ao aporte de nutrientes, e às indústrias.

\section{Índice final e ranqueamento do saneamento ambiental nas RHRJ}

Com relação ao Índice Final, a pontuação obtida na Figura 6 reflete os valores resultantes da integração dos resultados de todos os sub-índices aplicados para as categorias Cobertura, Eficiência, Demanda e Gestão. A melhor pontuação obtida foi para a RHRJ VI (Lagos São João), seguida da RHRJ III (Médio Paraíba do Sul) e a pior pontuação foi obtida pela RHRJ IX (Baixo Paraíba do Sul e Itabapoana). 


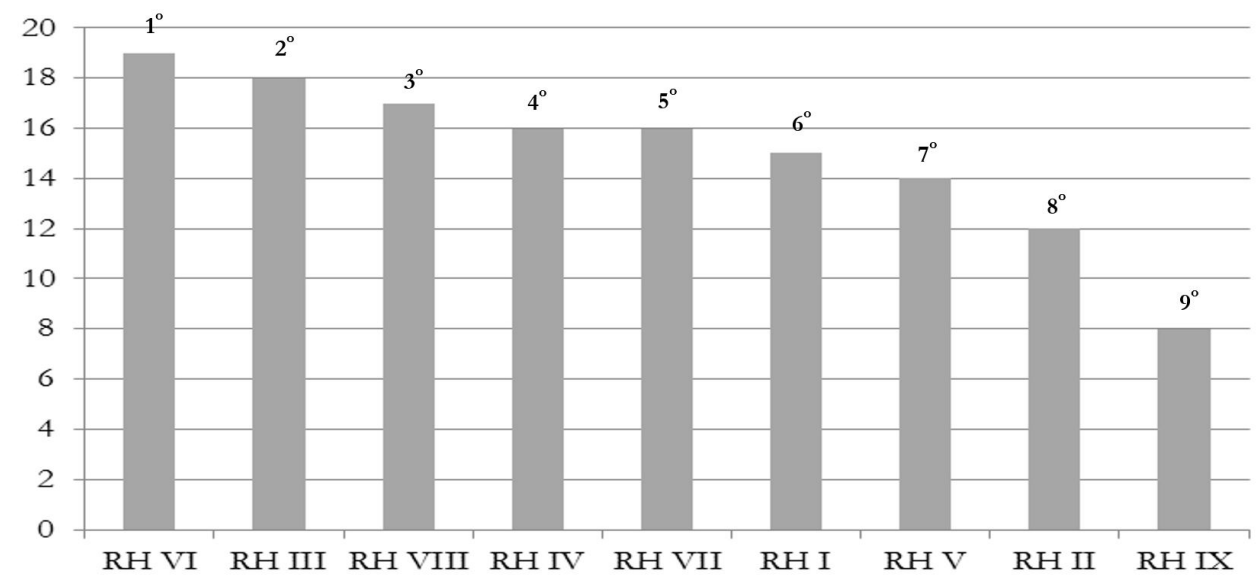

Figura 6. Índice final e ranqueamento do saneamento ambiental nas RHRJ.

Apesar dos resultados dos sub-índices variarem muito em uma mesma RHRJ, este resultado final pode ser explicado pelo fato da RHRJ e comitê VI (Lagos São João) terem avançado em relação à Gestão de recursos hídricos. Isto ocorreu a partir da implementação do Fundo de Boas Práticas Socioambientais em Microbacias -Funboas e do Consórcio Intermunicipal Lagos São João. O Funboas foi formado com um percentual do pagamento pelo uso da água da bacia, mas é aberto e pode receber contribuição de ONGs, empresas e outras fontes. Posteriormente, um projeto de Pagamento por Serviços Ambientais (PSA) hídrico, apoiado pela Agência Nacional de Águas, estabelecido na região tem dado continuidade nas ações em prol da conservação dos recursos hídricos nesta RHRJ.

Também quando se observa o Plano Estadual de Recursos Hídricos - PERHI (RIO DE JANEIRO, 2014), o rio São João foi o que apresentou menores índices de violação de classe 2 para todos os parâmetros analisados de qualidade da água, o que demonstra a eficiência no tratamento de esgoto.

Contudo, em termos de demanda pelo uso da água para abastecimento humano, segundo PERHI (RIO DE JANEIRO, 2014) as RHRJ V (Baia de Guanabara) e IX (Baixo Paraíba do Sul e Itabapoana) são as maiores demandantes, o que indica que precisam se planejar e tomar medidas de conservação e uso eficiente da água para mitigar a escassez de água futura. Ressalta-se ainda, que a RHRJ IX vem sofrendo as consequências da transposição de água do rio Paraíba do Sul para o rio Guandu, tais como elevada sedimentação do curso d'água, reduzindo sua capacidade de armazenamento de água, bem como a salinização da água, o que tem implicado na sua escassez para os diferentes usos múltiplos na RHRJ IX.

O PERHI (RIO DE JANEIRO, 2014) prevê diversas ações relacionadas à gestão de recursos hídricos para o estado do Rio de Janeiro, sendo prioritárias: construção de um pacto de gestão para a segurança hídrica no sistema Paraíba do Sul-Guandu; desenvolvimento do sistema de informações sobre recursos hídricos; comunicação na gestão dos recursos hídricos e melhorias dos sistemas de coleta e tratamento de esgoto.

\section{CONCLUSÕES}

De modo geral observou-se que não há uma tendência de uma RHRJ apresentar todos os sub-índices na mesma classe. A melhor pontuação obtida no ranqueamento final foi na RHRJ VI (Lagos São João), seguida da RHRJ III (Médio Paraíba do Sul) e a pior pontuação foi obtida pela RHRJ IX (Baixo Paraíba do Sul e Itabapoana).

O trabalho traz contribuições importantes por agrupar os sub-índices nas categorias: Cobertura, Eficiência, Demanda e Gestão e fornecer um panorama integrado da situação de saneamento ambiental nas RHRJ.

Considera-se que uma melhoria no saneamento ambiental das RHRJ passa por uma gestão e governança eficientes, havendo necessidade de investimentos não apenas voltados à coleta de esgoto e abastecimento de água, mas também pelo aumento da eficiência do tratamento de esgoto e água e redução de perdas de água pelos sistemas de abastecimento e desperdícios pela população, devendose levar em conta as pressões antrópicas, dentre elas a demanda e conflitos relacionados aos recursos hídricos nas RHRJ.

Contudo, observou-se que as referências ou metas para apoiar na classificação dos sub-índices no nível municipal e de RHRJ são frágeis e divergentes. Sugere-se para trabalhos futuros a agregação de outros índices relativos à qualidade da água, disposição de lixo e nível de atuação dos Comitês de Bacias na gestão de recursos hídricos, bem como níveis de investimentos no saneamento ambiental. 


\section{AGRADECIMENTO}

Os autores agradecem à bolsa concedida pelo projeto MP5 05.14.01.001.00.00, liderado por André Marcelo de Souza da Embrapa Solos.

\section{REFERÊNCIAS}

AQUINO, M. C. Você sabe a diferença entre saneamento ambiental e saneamento básico? Curitiba: Sinergia, 2018. Disponível em: <http://www.sinergiaengenharia.com.br/voce-sabe-a-diferenca-entre-saneamentoambiental-e-saneamento-basico/>. Acesso em: 1 abr. 2018.

ASSOCIAÇÃO PRÓ-GESTÃO DAS ÁGUAS DA BACIA HIDROGRÁFICA DO RIO PARAÍBA DO SUL - AGEVAP. Plano integrado de recursos hídricos da bacia hidrográfica do Rio Paraíba do Sul e planos de ação de recursos hídricos das bacias afluentes: diagnóstico das fontes de poluição. Resende: AGEVAP, 2014. 145 p.

BANCO MUNDIAL. Relatório anual de 2016. ONU Brasil, 2018. Disponível em: <https://nacoesunidas.org/bancomundial-america-latina-tem-agua-em-abundancia-mas-falta-saneamento/>. Acesso em: 1 abr. 2018.

BRAGA, T. M. et al. Índices de sustentabilidade municipal: o desafio de mensurar. Nova Economia, Belo Horizonte, v. 14, p. 11-33, 2004.

BRASIL. Ministério da Saúde. Portaria no 2.914, de 12 de dezembro de 2011. Dispõe sobre procedimentos de controle e de vigilância da qualidade da água para consumo humano e seu padrão de potabilidade. Diário Oficial [da] República Federativa do Brasil, Poder Executivo, Brasília, DF, 14 dez. 2011.

BRASIL. Ministério das Cidades. Plano Nacional de Saneamento Básico. Secretaria Nacional de Saneamento Ambiental. Brasília. DF: Ministério das Cidades 2013.

BRASIL. Ministério das Cidades. Secretaria Nacional de Saneamento Ambiental - SNSA. Sistema Nacional de Informações sobre Saneamento: diagnóstico dos serviços de água e esgotos 2013. Brasília: SNSA/MCIDADES, 2014. 181 p. Disponível em: <https://engineering.columbia.edu/files/engineering/design-waterresource07.pdf>. Acesso em: 1 abr. 2018.

CENTRO ESTADUAL DE ESTATÍSTICAS, PESQUISAS E FORMAÇÃO DE SERVIDORES PÚBLICOS DO RIO DE JANEIRO - CEPERJ. Centro de Estatísticas, Estudos e Pesquisas - CEEP. 2018. Disponível em: <http://www.ceperj.rj.gov.br/ceep/ceep.html>. Acesso em: 1 abr. 2018.

COSTA, S. A. B. et al. Indicadores em saneamento: avaliação da prestação dos serviços de água e de esgoto em Minas Gerais. Revista UFMG, Belo Horizonte, v. 20, n. 2, p. 334-357, 2013.

DIAS, F. A.; GOMES, L. A.; ALKMIM, J. K. Avaliação da qualidade ambiental urbana da bacia do Ribeirão do Lipa através de indicadores. Sociedade \& Natureza, Cuiabá, v. 23, p. 127-142, 2011.

GEOINEA. Base de dados geoespaciais. Instituto Estadual do Ambiente, 2019. Disponível em: <https://inea.maps.arcgis.com/apps/MapSeries/index.html?appid=00cc256c620a4393b3d04d2c34acd9ed >. Acesso em: 1 jan. 2019.

GIOVANNINI, E. et al. Handbook on constructing composite indicators: methodology and user guide. Paris: Organisation for Economic Co-operation and Development, 2008.

GONÇALVES, F. M. Bacia hidrográfica do rio Paraíba do Sul: avaliação integrada da qualidade das águas dos estados de Minas Gerais, Rio de Janeiro e São Paulo. 2016141 f. Tese (Doutorado) - Programa de Pósgraduação em Saneamento, Meio Ambiente e Recursos Hídricos, Universidade Federal de Minas Gerais, Belo Horizonte, 2016.

INSTITUTO TRATA BRASIL. Ranking do saneamento 2017. São Paulo, 2017. Disponível em: <http://www.tratabrasil.org.br/component/estudos/itb/ranking-do-saneamento-2017>. Acesso em: 1 maio 2019.

KURY, K. A. et al. A percepção ambiental da população do baixo Paraíba do Sul sobre a qualidade da água e estratégia de recuperação: resultados preliminares. In: SIMPÓSIO DE RECURSOS HÍDRICOS DA BACIA DO RIO PARAÍBA DO SUL, 1., 2008, Resende. Anais... Resende: CEIVAP, 2008.

LIMA, V. Saneamento ambiental como indicador de análise da qualidade ambiental urbana. Caderno Prudentino de Geografia, Presidente Prudente, v. 2, n. 35, p. 65-84, 2013.

MEIJERING, J. V.; KERN, K.; TOBI, H. Identifying the methodological characteristics of European green city rankings. Ecological Indicators, New York, v. 43, p. 132-142, 2014.

NIRAZAWA, A. N.; OLIVEIRA, S. V. W. B. Sanitation indicators: analysis of variables to construct municipality indicators. Revista de Administração Pública, Rio de Janeiro, v. 4, n. 52, p. 753-763, 2018.

ORGANISATION FOR ECONOMIC COOPERATION AND DEVELOPMENT - OECD. Environmental indicators: development, measurement and use: reference paper. Paris, 1993. 37 p. Disponível em: <https://www.oecd.org/env/indicators-modelling-outlooks/24993546.pdf>. Acesso em: 1 abr. 2018.

ORGANIZAÇÃO DAS NAÇÕES UNIDAS - ONU. International Decade for Action Water for Life 2005-2015, 2015. Disponível em: <http://www.un.org/waterforlifedecade/food_security.shtml>. Acesso em: 1 de abril 2018. 
PIMENTEL, L. B. et al. Saneamento | 0 apoio do BNDES ao saneamento no âmbito do Programa de Aceleração do Crescimento. BNDES Setorial, Rio de Janeiro, v. 45, p. 227-284, 2017.

QUEIROZ, V. C.; NASCIMENTO, N. O. Estimativa do déficit de investimentos em saneamento em Minas Gerais. Revista de Gestão de Água da América Latina, Porto Alegre, v. 1, n. 2, p. 186-197, 2016.

RIO DE JANEIRO. Instituto Estadual do Ambiente. Decreto n. 42.930, de 18 de abril de 2011. Cria o programa estadual pacto pelo saneamento. Diário Oficial, Rio de Janeiro, RJ, 19 abr. 2011.

RIO DE JANEIRO. Instituto Estadual do Ambiente - INEA. Resolução do Conselho Estadual de Recursos Hídricos $n^{\circ}$ 107, de 22 de maio de 2013. Diário Oficial, Rio de Janeiro, RJ, 12 jun. 2013. Disponível em: <http://www.ceivap.org.br/legirj/ResolucoesCERHI/Resolucao-CERHI\%20107.pdf>. Acesso em: 1 abr. 2018.

RIO DE JANEIRO. Secretaria de Estado do Ambiente - SEA. Instituto Estadual do Ambiente - INEA. Plano estadual de recursos hídricos do Estado do Rio de Janeiro: relatório diagnóstico, versão final. Rio de Janeiro, 2014. 378 p. Disponível em:

<http://www.inea.rj.gov.br/cs/groups/public/documents/document/zwew/mdyy/ edisp/inea0062195. pdf >. Acesso em: 1 abr. 2018.

RUFINO, R. C. Avaliação da qualidade ambiental do município de Tubarão (SC) através do uso de indicadores ambientais. 2002123 f. Dissertação (Mestrado em Engenharia de Produção) - Departamento de Engenharia de Produção, Universidade Federal de Santa Catarina, Florianópolis, 2005.

SÃO PAULO. Secretaria do Meio Ambiente - SMA. Município VerdeAzul. São Paulo, 2019. Disponível em: <www.ambiente.sp.gov.br/municipioverdeazul/>. Acesso em: 1 maio 2019.

SCHWEMLEIN, S.; CRONK, R.; BARTRAM, J. Indicators for monitoring water, sanitation, and hygiene: a systematic review of indicator selection methods. International Journal of Environmental Research and Public Health, Basel, v. 13, n. 3, p. 1-15, 2016.

SOUZA, C. M. N. S. et al. Saneamento: promoção da saúde, qualidade de vida e sustentabilidade ambiental. Rio de Janeiro: Fiocruz, 2015. 139 p.

SPERLING, T. L.; SPERLING, M. Proposição de um sistema de indicadores de desempenho para avaliação da qualidade dos serviços de esgotamento s anitário. Engenharia Sanitaria e Ambiental, Rio de Janeiro, v. 18, n. 4, p. 313-322, 2013.

TEIXEIRA, J. C. et al. Estudo do impacto das deficiências de saneamento básico sobre a saúde pública no Brasil no período de 2001 a 2009. Engenharia Sanitaria e Ambiental, Rio de Janeiro, v. 19, n. 1, p. 87-96, 2014.

TOMELERI, D. B.; CAMPOS, T. R.; MORETE, V. S. Saneamento ambiental e sustentabilidade: essencialidade à vida humana e à proteção do meio ambiente. Revista de Ciências Jurídicas, Maringá, v. 14, n. 2, p. 233-241, 2013.

TUCCI, C. E. M. Indicador de sustentabilidade hídrica urbana. Revista de Gestão de Água da América Latina, Porto Alegre, v. 14, e7, 2017.

VAN BELLEN, H. M. Indicadores de sustentabilidade: um levantamento dos principais sistemas de avaliação. Cadernos EBAPE.BR, Rio de Janeiro, v. 2, n. 1, p. 1-14, 2004.

\section{Contribuições dos autores}

Tatiana Lourenço: Contribuiu com a obtenção e organização da base de dados, com a composição e aplicação dos índices (para os municípios e regiões hidrográficas do estado do Rio de Janeiro) e com a elaboração do artigo.

Rachel Bardy Prado: Contribuiu com a definição da metodologia a ser aplicada, com a composição dos índices (para os municípios e regiões hidrográficas do estado do Rio de Janeiro), com a revisão da literatura e com a elaboração do artigo. 